\title{
XLV. On the propagation of laminar motion through a turbulently moving inviscid liquid
}

\section{Sir William Thomson LL.D. F.R.S.}

To cite this article: Sir William Thomson LL.D. F.R.S. (1887) XLV. On the propagation of laminar motion through a turbulently moving inviscid liquid, Philosophical Magazine Series 5, 24:149, 342-353, DOI: $10.1080 / 14786448708628110$

To link to this article: http://dx.doi.org/10.1080/14786448708628110

册 Published online: 29 Apr 2009.

Submit your article to this journal $\lceil\pi$

山 Article views: 11

Q View related articles $\asymp$ 
power of) error, abstraction faite du signe, is

$$
\text { twice (Mean-square-of-error-(Mean-Error) }{ }^{2} \text { ) }
$$

that is, here,

$$
\text { Number of Observations }
$$

$$
2(6 \cdot 4-4) \div 25878=\cdot 00019 \text {. }
$$

Whence the Modulus is about $\cdot 014$, while the observed écart is 4 - some thirty times larger. This gives odds of nonillions to one against the First Exponential. The corresponding odds against the Probability-curve are some hundreds to one.

It should be observed that the sums of powers may be taken for integral portions of the curve's extent, rather than for the whole. This plan seems theoretically more correct, since the fulfilment of the law of error is to be looked for rather in the body of the curve than at the extremities. There arise, however, practical difficulties about the computation in the case of some curves; in the absence of tables for the values of $\int x^{2} \phi(x) d x$. The Probability-curve itself affords an instance.

Different modes of verification will be appropriate to different cases. But it is not the purpose of this paper to provide a complete Manual of empirical evidence; but rather to show in what sort of way the examination of experience may be assisted by the Mathematical Theory of Errors and Method of Statistics.

XLV. On the Propagation of Laminar Motion through a turbulently moving Inviscid Liquid. By Sir William Thomson, LL.D., F.R.S.*

1. T $\mathrm{N}$ endeavouring to investigate turbulent motion of water 1 between two fixed planes, for a promised communication to Section A of the British Association at its coming Meeting in Manchester, I have found something seemingly towards a solution (many times tried for within the last twenty years) of the problem to construct, by giving vortex motion to an incompressible inviscid fluid, a medium which shall transmit waves of laminar motion as the luminiferous æther transmits waves of light.

2. Let the fluid be unbounded on all sides, and let $u, v, w$ be the velocity-components, and $p$ the pressure at $(x, y ; z, t)$. We have

$$
\frac{d u}{d x}+\frac{d v}{d y}+\frac{d w}{d z}=0 \quad \text {. . . . . }
$$

* Communicated by the Author, having been read before Section A of the British Association at its recent Meeting in Manchester. 
Motion through a turbulently moving Inviscid Liquid. 343

$$
\begin{aligned}
& \frac{d u}{d t}=-\left(u \frac{d u}{d x}+v \frac{d u}{d y}+w \frac{d u}{d z}+\frac{d p}{d x}\right) \\
& \frac{d v}{d t}=-\left(u \frac{d v}{d x}+v \frac{d v}{d y}+w \frac{d v}{d z}+\frac{d p}{d y}\right) \\
& \frac{d w}{d t}=-\left(u \frac{d w}{d x}+v \frac{d w}{d y}+w \frac{d w}{d z}+\frac{d p}{d z}\right) \ldots
\end{aligned}
$$

From (2), (3), (4) we find, taking (1) into account,

$$
-\nabla^{2} p=\frac{d u^{2}}{d x^{2}}+\frac{d v^{2}}{d y^{2}}+\frac{d w^{2}}{d z^{2}}+2\left(\frac{d v}{d z} \frac{d w}{d y}+\frac{d w}{d x} \frac{d u}{d z}+\frac{d u}{d y} \frac{d v}{d z}\right) \text {. }
$$

3. The velocity-components $u, v, w$ may have any values whatever through all space, subject only to (1). Hence, on Fourier's principles, we have, as a perfectly comprehensive expression for the motion at any instant,

$$
\begin{aligned}
& u=\Sigma \Sigma \Sigma \Sigma \Sigma \Sigma \alpha_{(m, n, q)}^{(e, f, g)} \sin (m x+e) \cos (n y+f) \cos (q z+g) \cdot(6), \\
& v=\Sigma \Sigma \Sigma \Sigma \Sigma \Sigma \beta_{(m, n, q)}^{(e, f, g)} \cos (m x+e) \sin (n y+f) \cos (q z+g) \cdot(7), \\
& w=\Sigma \Sigma \Sigma \Sigma \Sigma \Sigma \gamma_{(m, n, q)}^{(e, f, g)} \cos (m x+e) \cos (n y+f) \sin (q z+g) \cdot(8)
\end{aligned}
$$

where $\alpha_{(m, n, q)}^{(e, f, g)}, \beta_{(m, n, q)}^{(e, f, g)}, \gamma_{(m, n, q)}^{(e, f, g)}$ are any three velocities satisfying the equation

$$
0=m \alpha_{(m, n, q)}^{(e, f, g)}+n \beta_{(m, n, q)}^{(e, f, g)}+q \gamma_{(m, n, q)}^{(e, f, g)} \quad . \quad . .
$$

and $\Sigma \Sigma \Sigma \Sigma \Sigma \Sigma$ summation (or integration) for different values of $m, n, q, e, f, g$. The summations for $e, f, g$ may, without loss of generality, be each confined to two values : $e=0$, and $e=\frac{1}{2} \pi ; f=0$, and $f=\frac{1}{2} \pi ; g=0$, and $g=\frac{1}{2} \pi$. We shall admit large values, and infinite values of $m^{-1}, n^{-1}, q^{-1}$, under certain conditions $[\$ 4(10),(11),(12)$, and $\$ 15$ below], but otherwise we shall suppose the greatest value of each of them to be of some moderate, or exceedingly small, linear magnitude. This is an essential of the averagings to which we now proceed.

4. Let xav, xzav, xyzav denote space-averages, linear, surface, and solid, through infinitely great spaces, defined and illnstrated by examples, each worked out from (6), (7), (8), as follows, $\mathrm{L}$ denoting an infinitely great length, or a very great multiple of whichever of $m^{-1}, n^{-1}, q^{-1}$ may be concerned :- 
344 Sir W. Thomson on the Propagation of Laminar

$$
\begin{aligned}
\operatorname{xav} u & =\frac{1}{2 \mathrm{~L}} \int_{-\mathrm{L}}^{\mathrm{L}} d x u=\Sigma \Sigma \Sigma \Sigma\left(\alpha_{(0, n, q)}^{\left(\frac{1}{2} \pi, f, g\right)} \cos (n y+f) \cos (q z+g) \cdot(10),\right. \\
\operatorname{xzav} u & =\left(\frac{1}{2 \mathrm{~L}}\right)^{2} \int_{-\mathrm{L}}^{\mathrm{L}} \int_{-\mathrm{L}}^{\mathrm{L}} d z d x u=\sum_{n}^{f} \sum_{(0, n, 0)}^{\left(\frac{1}{2} \pi, f, 0\right)} \cos (n y+f) \cdot(11), \\
\operatorname{xyzav} u & =\left(\frac{1}{2 \mathrm{~L}}\right)^{3} \int_{-\mathrm{L}}^{\mathrm{L}} \int_{-\mathrm{L}}^{\mathrm{L}} \int_{-\mathrm{L}}^{\mathrm{L}} d z d y d x u=\alpha_{(0,0,0)}^{\left(\frac{1}{2} \pi, 0,0\right)} \cdot(12), \\
\operatorname{xav} u^{2} & =\frac{1}{2} \Sigma \Sigma \Sigma \Sigma \Sigma \Sigma\left[\alpha_{(m, n, q)}^{(e, f, g)}\right] \cos ^{2}(n y+f) \cos ^{2}(q z+g) \cdot(13) ;
\end{aligned}
$$

this with the exceptions that

in the case of $m=0, e=0$, we take 0 in place of $\frac{1}{2}$, and in the case of $m=0, e=\frac{1}{2} \pi \quad, 1 \quad$ " , .

$$
\begin{aligned}
\text { xzav } u^{2} & =\frac{1}{4} \sum \sum_{m}^{e} \sum_{m}^{f} \sum_{m}^{g} \sum \sum_{(m, n, q)}\left[\alpha^{(e, f, g)}\right]^{2} \cos ^{2}(n y+f) \\
\operatorname{xzav} u v & =\frac{1}{4} \sum_{\sum}^{f} \sum_{m n}^{g} \sum \sum_{q}\left[\alpha_{(m, n, q)}^{\left(\frac{1}{2} \pi, f, g\right)} \beta_{(m, n, q)}^{(0, f, g)}\right. \\
& \left.-\alpha_{(m, n, q)}^{(0, f, g)} \beta_{(m, n, q)}^{\left(\frac{1}{2} \pi, f, g\right)}\right] \cos (n y+f) \sin (n y+f) .
\end{aligned}
$$

with the exceptions for (14) that

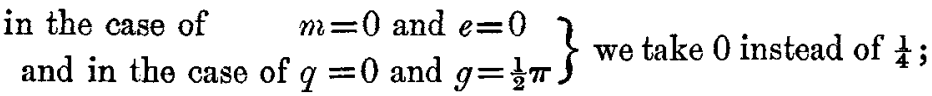
in the case of $\left.\begin{array}{l}m=0 \text { and } e=\frac{1}{2} \pi \\ q=0 \text { and } g=0\end{array}\right\}$ and in the case of $q=0$ and $g=0\} \quad, \quad \frac{1}{2} \quad, \quad, \frac{1}{4}$; in the case of $m=0, e=\frac{1}{2} \pi, n=0, f=\frac{1}{2} \pi, 1, \quad$, $\quad \frac{1}{4}$; and analogous exceptions for (15).

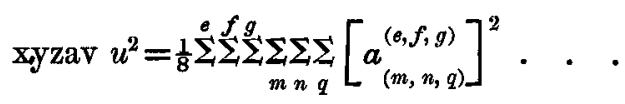

with exceptions for zeros of $m$ and $q$, analogous to those of (14). 5. As a last example of averagings for the present, take xyzav of (5). Thus we find

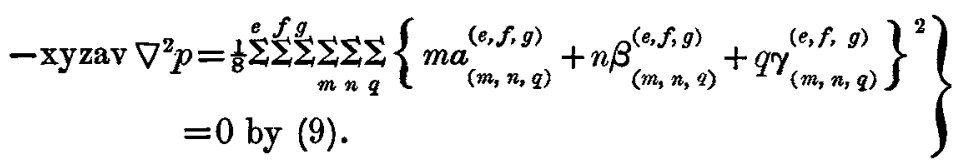

The interpretation is obvious. 
6. Remark, as a general property of this kind of averaging,

$$
\operatorname{xav} \frac{d Q}{d x}=0 \quad \text {. . . . . . . }
$$

if $Q$ be any quantity which is finite for infinitely great values of $x$.

7. Suppose now the motion to be homogeneously distributed through all space. This implies that the centres of inertia of all great volumes of the fluid have equal parallel motions, if any motions at all. Conveniently, therefore, we take our reference lines $O X, O Y, O Z$, as fixed relatively to the centres of inertia of three (and therefore of all) centres of inertia of large volumes ; in other words, we assume no translatory motion of the fluid as a whole. This makes zero of every large average of $u$ and of $v$ and of $w$; and, in passing, we may remark, with reference to our notation of $\S 3$, that it makes, as we see by $(10),(11),(12)$,

$$
0=\alpha_{(0, n, q)}=\alpha_{(m, 0, q)}=\alpha_{(m, n, 0)}=\beta_{(0, n, q)}=\& \mathrm{c} . \& \mathrm{c} .=\gamma_{(m, n, 0)}
$$

Without for the present, however, encumbering ourselves with the Fourier-expression and notation of $\S 3$, we may write, as the general expression for nullity of translational movement in large volumes,

$$
0=\text { ave } u=\text { ave } v=\text { ave } w \text {. . . . }
$$

where ave denotes the average through any great length of straight or curved line, or area of plane or curved surface, or through any great volume of space.

8. In terms of this generalized notation of averages, homogeneousness implies

$$
\begin{array}{lll}
\text { ave } u^{2}=\mathrm{U}^{2}, & \text { ave } v^{2}=\mathrm{V}^{2}, & \text { ave } w^{2}=\mathrm{W}^{2} . \\
\text { ave } v w=\mathrm{A}^{2}, & \text { ave } w u=\mathrm{B}^{2}, & \text { ave } u v=\mathrm{C}^{2} .
\end{array}
$$

where $\mathrm{U}, \mathrm{V}, \mathrm{W}, \mathrm{A}, \mathrm{B}, \mathrm{C}$ are six velocities independent of the positions of the spaces in which the averages are taken. These equations are, however, infinitely short of implying, though implied by, homogeneousness.

9. Suppose now the distribution of motion to be isotropic. This implies, but is infinitely more than is implied by, the following equations in terms of the notation of $\S 8$, with further notation, $R$, to denote what we shall call THE AVERAGE VELOCITY of the turbulent motion :-

$$
\begin{aligned}
& \mathrm{U}^{2}=\mathrm{V}^{2}=\mathrm{W}^{2}=\frac{1}{3} \mathrm{R}^{2} \quad . \quad . \quad . \quad(23), \\
& 0=\mathrm{A}=\mathrm{B}=\mathrm{C} \text {. . . . . . . (24). }
\end{aligned}
$$

10. Large questions now present themselves as to transPhil. Mag. S. 5. Vol. 24. No. 149. Oct. 1887. 2 A 
formations which the distribution of turbulent motion will experience in an infinite liquid left to itself with any distribution given to it initially. If the initial distribution be homogeneous through all large volumes of space, except a certain large finite space, $\mathrm{S}$, through which there is initially either no motion, or turbulent motion homogeneous or not, but not homogeneous with the motion through the surrounding space, will the fluid which at any time is within $S$ acquire more and more nearly as time advances the same homogeneous distribution of motion as that of the surrounding space, till ultimately the motion is homogeneous throughout?

11. If the answer were yes, could it be that this equalization would come to pass through smaller and smaller spaces as time advances? In other words, would any given distribution, homogeneous on a large enough scale, become more and more fine-grained as time advances? Probably yes for some initial distributions ; probably no for others. Probably yes for vortex motion given continuously through all of one large portion of the fluid, while all the rest is irrotational.

12. Probably no for the initial motion given in the shape of equal and similar Helmholtz rings, of proportions suitable for individual stability, and each of overall diameter considerably smaller than the average distance from nearest neighbour. Prodably also no, though the rings be of very different volumes and vorticities. But probably yes if the diameters of the rings, or of many of them, be not small in comparison with distances from neighbours, or if the individual rings, each an endless slender filament, be entangled or nearly entangled among one another.

13. Again a question : If the initial distribution be homogeneous and coolotropic, will it become more and more isotropic as time advances, and ultimately quite isotropic? Probably yes, for any random initial distribution, whether of continuous rotationally-moving fluid or of separate finite vortex rings. Possibly no for some symmetrical initial distribution of vortex rings, conceivably stable.

14 If the initial distribution be homogeneous and isotropic (and therefore utterly random in respect to direction), will it remain so? Certainly yes. I proceed to investigate a mathematical formula, deducible from the answer, which will be of use to us later ( $\$ 18)$. By (22) and (24) we have

$$
\text { xzav } u v=0 \text {, for all values of } t \quad \cdot \quad \cdot \quad \text { (25). }
$$

But by (2) and (3) we find

$\frac{d}{d t}(\operatorname{xzav} u v)=-\mathrm{xza}\left\{u \frac{d(u v)}{d x}+v \frac{d(u v)}{d y}+w \frac{d(u v)}{d z}+v \frac{d p}{d x}+u \frac{d p}{d y}\right\}$ 
Hence

$$
0=\operatorname{xzav}\left\{u \frac{d(u v)}{d x}+v \frac{d(u v)}{d y}+w \frac{d(u v)}{d z}+v \frac{d p}{d x}+u \frac{d p}{d y}\right\} .
$$

This equation in fact holds for every random case of motion satisfying (30) below, because positive and negative values of $u, v, w$ are all equally probable, and therefore the value of the second member of (27) is doubled by adding to itself what it becomes when for $u, v$, we substitute $-u,-v,-w$, which, it may be remarked, and verified by looking at (5), does not change the value of $p$.

15. We shall now suppose the initial motion to consist of a laminar motion $[f(y), 0,0]$ superimposed on a homogeneous and isotropic distribution $\left(\mathfrak{u}_{0}, v_{0}, w_{0}\right)$; so that we have

$$
\text { when } t=0, \quad u=f(y)+u_{0}, \quad v=v_{0}, \quad w=w_{0} \quad . \quad(28) \text {; }
$$

and we shall endeavour to find such a function, $f(y, t)$, that at any time $t$ the velocity-components shall be

$$
f(y, t)+\mathfrak{l}, v, w \quad \text {. . . . . }
$$

where $\mathfrak{u}, v, w$ are quantities of each of which every large enough average is zero, so that particularly, for example,

$$
0=\operatorname{xzav} \mathfrak{u}=\operatorname{xzav} v=\operatorname{xzav} w \quad . \quad \text {. }(30) .
$$

16. Substituting (29) for $u, v, w$ in (2) we find

$$
\frac{d f(y, t)}{d t}+\frac{d \mathfrak{u}}{d t}=-\left\{f(y, t) \frac{d \mathfrak{u}}{d x}+v \frac{d f(y, t)}{d y}\right\}-\left(\mathfrak{u} \frac{d \mathfrak{u}}{d x}+v \frac{d \mathfrak{u}}{d y}+w \frac{d \mathfrak{u}}{d z}+\frac{d p}{d x}\right)
$$

Take now xzav of both members. The second term of the first member and the second term of the second member disappear, each in virtue of (30). The first and last terms of the second member disappear, each in virtue of (18) alone and also each in virtue of $(30)$. There remains

$$
\frac{d f(y, t)}{d t}=-\operatorname{xzav}\left(\mathfrak{u} \frac{d \mathfrak{u}}{d x}+v \frac{d \mathfrak{u}}{d y}+w \frac{d \mathfrak{l}}{d z}\right) .
$$

To simplify, add to the second member [by (1)]

$$
0=-\operatorname{xzav}\left(\mathfrak{u} \frac{d \mathfrak{u}}{d x}+\mathfrak{u} \frac{d v}{d y}+\mathfrak{u} \frac{d w}{d z}\right) . . . .
$$

and, the first and third pair of terms of the thus-modified second member vanishing by (18), find

$$
\frac{d f(y, t)}{d t}=-\operatorname{xzav} \frac{d(\mathfrak{u} v)}{d y} \text {. . . . . }
$$

It is to be remarked that this result involves, besides (1), 2 A 2 
no other condition respecting ( $\mathfrak{u}, v, w)$ than (30); no isotropy, no homogeneousness in respect to $y$; and only homogeneousness of régime with respect to $y$ and $z$, with no mean translational motion.

The $x$-translational mean component of the motion is wholly represented by $f(y, t)$; and, so far as our establishment of (34) is concerned, may be of any magnitude, great or small relatively to velocity-components of the turbulent motion. It is a fundamental formula in the theory of the turbulent motion of water between two planes; and I had found it in endeavouring to treat mathematically my brother Prof. James Thomson's theory of the "Flow of Water in Uniform Régime in Rivers and other Open Channels" *. In endeavouring to advance a step towards the law of distribution of the laminar motion at different depths, I was surprised to discover the seeming possibility of a law of propagation as of distortional waves in an elastic solid, which constitutes the conclusion of my present communication, on the supposition of $\S 15$ that the distribution $\mathfrak{t}_{0}, v_{0}, w_{0}$ is isotropic, and that $d f(y, t) / d y$, divided by the greatest value of $f(y, t)$, is infinitely small in comparison with the smallest values of $m, n, q$, in the Fourier-formulæ (6), (7), (8) for the turbulent motion.

17. By (34) we see that, if the turbulent motion remained, through time, isotropic as at the beginning, $f(y, t)$ would remain constantly at its initial value $f(\dot{y})$. To find whether the turbulent motion does remain isotropic, and, if it does not, to find what we want to know of its deviation from isotropy, let us find $\operatorname{xzav} \frac{d(\boldsymbol{u} v)}{d t}$, by (2) and (3), as follows:First, by multiplying (31) by $v$, and (3) by $\mathfrak{u}$, and adding, we find

$$
\begin{aligned}
v \frac{d f(y, t)}{d t} & +\frac{d(\mathfrak{u} v)}{d t}=-\left\{f(y, t) \frac{d(\mathfrak{u} v)}{d x}+v^{2} \frac{d f(y, t)}{d y}\right\} \\
& -\left\{\mathfrak{u} \frac{d(\mathfrak{u} v)}{d x}+v \frac{d(\mathfrak{u} v)}{d y}+w \frac{d(\mathfrak{u} v)}{d z}+v \frac{d p}{d x}+\mathfrak{u} \frac{d p}{d y}\right\} .
\end{aligned}
$$

Taking xzav of this, and remarking that the first term of the first member disappears by (30), and the first term of the second member by (18), we find, with $\mathrm{V}^{2}$, as in $\$ \S 8,9$, to denote the average $y$-component-velocity of the turbulent motion,

$$
\frac{d}{d t}\{\operatorname{xzav}(\mathfrak{u} \bar{v})\}=-\nabla^{2} \frac{d f(y, t)}{d y}-\mathrm{Q} . .
$$

* Proceedings of the Royal Society, Aug. 15, 1878. 
Motion through a turbulently moving Inviscid Liquid. 349 where

$$
\mathrm{Q}=\mathrm{xzav}\left\{\mathfrak{u} \frac{d(\mathfrak{u} v)}{d x}+v \frac{d(\mathfrak{u} v)}{d y}+w \frac{d(\mathfrak{u} v)}{d z}+v \frac{d p}{d x}+\mathfrak{u} \frac{d p}{d y}\right\} .
$$

18. Let

$$
p=\mathfrak{p}+\varpi
$$

where $\mathfrak{p}$ denotes what $p$ would be if $f$ were zero. We find, by (5),

and, by (27) and (37),

$$
-\nabla^{2} \varpi=2 \frac{d f(y, t)}{d y} \frac{d v}{d x} \cdot \ldots . .
$$

$$
\mathrm{Q}=\operatorname{xzav}\left(v \frac{d \varpi}{d x}+\mathfrak{u} \frac{d \varpi}{d y}\right) ; . . . .
$$

So far we have not used either the supposition of initial isotropy for the turbulent motion, or of the infinitesimalness of $d f / d y$. We now must introduce and use both suppositions.

19. To facilitate the integration of (39), we now use our supposition that $\frac{d}{d t} f(y, t)$, divided by the greatest value of $f(y, t)$, is infinitely small in comparison with $m, n, q$, which, as is easily proved, gives

by which (40) becomes

$$
\varpi=2 \frac{d f(y, t)}{d y} \frac{1}{-\nabla^{2}} \frac{d v}{d x} \text {. . . . . . }
$$

$$
\mathrm{Q}=-2 \frac{d f(y, t)}{d y} \operatorname{xzav}\left(v \frac{d}{d x}+\mathfrak{u} \frac{d}{d y}\right) \nabla^{-2} \frac{d v}{d x} .
$$

Now, by $(x, z)$ isotropy, we have

$$
\begin{aligned}
& 2 \operatorname{xzav}\left(v_{0} \frac{d}{d x}+\mathfrak{u}_{0} \frac{d}{d y}\right) \nabla^{-2} \frac{d v_{0}}{d x} \\
& =\operatorname{xzav}\left\{v_{0}\left(\begin{array}{c}
d^{2} \\
d x^{2}
\end{array}+\frac{d^{2}}{d z^{2}}\right)+\left(\mathfrak{u}_{0} \frac{d}{d x}+w_{0} \frac{d}{d z}\right) \frac{d}{d y}\right\} \nabla^{-2} v_{0}
\end{aligned}
$$

Performing integrations by parts for the last two terms of the second member, and using (1), we find

$$
\begin{aligned}
\operatorname{xzav}\left(\mathfrak{u}_{0} \frac{d}{d x}+w_{0} \frac{d}{d z}\right) \frac{d}{d y} \nabla^{-2} v_{0} & =-\operatorname{xzav}\left(\frac{d \mathfrak{u}_{0}}{d x}+\frac{d w_{0}}{d z}\right) \frac{d}{d y} \nabla^{-2} v_{0} \\
& =\operatorname{xzav} \frac{d v_{0}}{d y} \frac{d}{d y} \nabla^{-2} v_{0} ;
\end{aligned}
$$

and so we find, by (43) and (42),

$$
\mathrm{Q}_{0}=-\frac{d f(y, t)}{d y} \operatorname{xzav}\left\{v_{0}\left(\frac{d^{2}}{d x^{2}}+\frac{d^{2}}{d z^{2}}\right)+\frac{d v_{0}}{d y} \frac{d}{d y}\right\} \nabla^{-2} v_{0}
$$


20. Using now the Fourier expansion (7) for $v_{0}$, we find $-\nabla^{-2} v_{0}=\sum_{\Sigma}^{e} \sum^{f} \sum_{m}^{E} \Sigma \sum \Sigma \beta_{(m, n, q)}^{(e, f, g)} \frac{\cos (m x+e) \sin (n y+f) \cos (q z+g)}{m^{2}+n^{2}+q^{2}}$.

Hence we find (with suffixes \&c. dropped),

and

$$
\operatorname{xzav} \frac{d v_{0}}{d y} \frac{d}{d y} \nabla^{-2} v_{0}=-\frac{1}{8} \Sigma \Sigma \Sigma \Sigma \Sigma \Sigma \frac{n^{2} \beta^{2}}{m^{2}+n^{2}+q^{2}}
$$

$$
\operatorname{xzav} v_{0}\left(\frac{d^{2}}{d x^{2}}+\frac{d^{2}}{d \tilde{z}^{2}}\right) \nabla^{-2} v_{0}=\frac{1}{8} \Sigma \Sigma \Sigma \Sigma \Sigma \Sigma \Sigma \Sigma \frac{\left(m^{2}+q^{2}\right) \beta^{2}}{m^{2}+n^{2}+q^{2}} \text {. }
$$

Now, in virtue of the average uniformity of the constituent terms implied in isotropy and homogeneousness $(\$ \S 7,8,9)$, the second member of (46) is equal to $-\frac{1}{8} \Sigma \Sigma \Sigma \Sigma \Sigma \Sigma \frac{\beta^{2}}{3}$, and therefore $(\S 9)$ equal to $-\frac{1}{9} R^{2}$; and similarly we see that the second member of (47) is equal to $+{ }_{9}^{2} R^{2}$. Hence, finally, by (44),

$$
\mathrm{Q}_{0}=-\frac{1}{9} \mathrm{R}^{2} \frac{d f(y, t)}{d y}
$$

and (36) for $t=0$, with $\frac{1}{3} \mathrm{R}^{2}$ for $V^{2}$ on account of isotropy, becomes

$$
\left\{\frac{d}{d t} \operatorname{xzav}(\mathfrak{t} w)\right\}_{t=0}=-\frac{2}{9} \mathrm{R}^{2}\left\{\frac{d f(y, t)}{d y}\right\}_{t=0} .
$$

The deviation from isotropy, which this equation shows, is very small, because of the smallness of $d f / d y$; and (27) does not need isotropy, but holds in virtue of (30). Hence (49) is not confined to the initial values (values for $t=0$ ) of the two members, because we neglect an infinitesimal deviation from ${ }_{9}^{2} \mathrm{R}^{2}$ in the first factor of the second member, considering the smallness of the second factor. Hence, for all values of $t$, unless so far as the "random" character referred to at the end of $\S 13$ may be lost by a rearrangement of vortices vitiating (27),

$$
\frac{d}{d t} \operatorname{xzav}(\mathfrak{t} w)=-2 \mathrm{R}^{2} \frac{d f(y, t)}{d y} . . . .
$$

21. Eliminating the first member from this equation, by (34), we find

$$
\frac{d^{2} f}{d t^{2}}=\frac{2}{9} R^{2} \frac{d^{2} f}{d y^{2}}
$$

Thus we have the very remarkable result that laminar disturbance is propagated according to the well-known mode of waves of distortion in a homogeneous elastic solid; and that the velocity of propagation is $\frac{\sqrt{ } 2}{3} R$, or about $\cdot 47$ of the

* Here and henceforth an averaging through $y$-spaces so small as to cover no sensible differences of $f(y, t)$, but infinitely large in proportion to
$n-1$, is implied. 
average velocity of the turbulent motion of the fluid. This might seem to go far towards giving probability to the vortex theory of the luminiferous ether, were it not for the doubtful proviso at the end of $\S 20$.

22. If the undisturbed condition of the medium be a stable symmetrical distribution of vortex-rings the suggested vitiation by "rearrangement" cannot occur. For example, let it be such as is represented in fig. 1, where the small white and

Fig. 1.

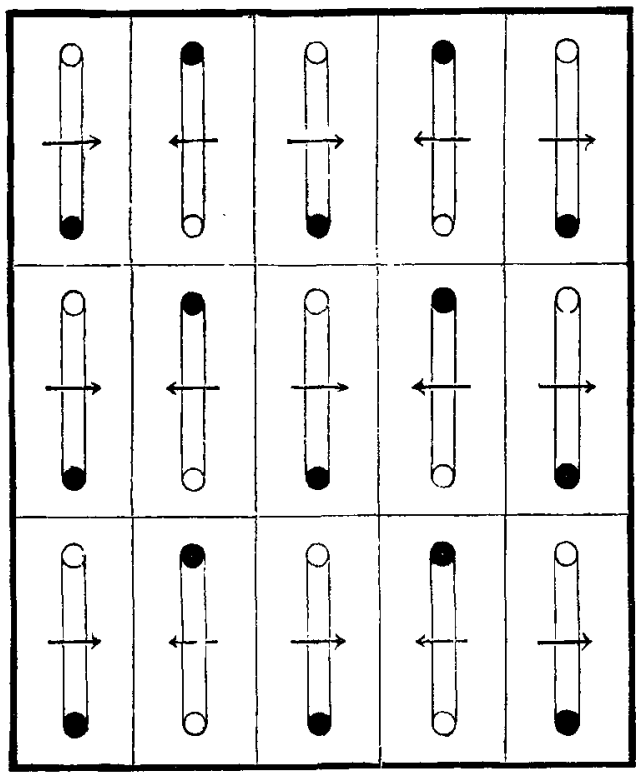

black circles represent cross sections of the rings : the white where the rotation is opposite to, and the black where it is in the same direction as, the rotation of the hands of a watch placed on the diagram facing towards the spectator. Imagine first each vortex-ring to be in a portion of the fluid contained within a rigid rectangular box, of which four sides are indicated by the fine lines crossing one another at right angles throughout the diagram; and the other pair are parallel to the paper, at any distance asunder we like to imagine. Supposing the volume of rotationally moving portion of the fluid constituting the ring to be given, there is clearly one determinate shape, and diametral magnitude, in which it must be given in order that the motion may be steady. Let it be so given, and fill space with such rectangular boxes of vortices arranged facing one another oppositely in the manner shown 
in the diagram. Annul now the rigidity of the sides of the boxes. The motion continues unchangedly steady. But is it stable, now that the rigid partitions are done away with? No proof has yet been given that it is. If it is, laminar waves, such as waves of light, could be propagated through it ; and the velocity of propagation would be $R \vee 2 / 3$ if the sides of the ideal boxes parallel to the undisturbed planes of the rings are square (which makes ave $\mathfrak{\imath}^{2}=$ ave $w^{2}$ ), and if the distance between the square sides of each box bears the proper ratio to the side of the square to make ave $v^{2}=$ ave $\mathfrak{u}^{2}=$ ave $w^{2}$.

23. Consider now, for example, plane waves, or laminar vibrations, in planes perpendicular to the undisturbed planes of the rings. The change of configuration of the vortices in the course of a quarter period of a harmonic standing vibration, $f(y, t)=\sin \omega t \cos \kappa g$ (which is more easily illustrated diagrammatically than a wave or succession of waves), is illustrated in fig. 2, for a portion of the fluid on each side of $y=0$. The upper part of the diagram represents the state of affairs when $t=0$; the lower when $t=\pi /(2 \omega)$. But it must not be overlooked, that all this $\$ \S 22,23$ depends on the unproved assumption that the symmetrical arrangement is stable.

24. It is exceedingly doubtful, so far as I can judge after much anxious consideration from time to time during these last twenty years, whether the configuration represented in fig. 1, or any other symmetrical arrangement, is stable when the rigidity of the ideal partitions enclosing each ring separately is annulled throughout space. It is possible that the rigidity of two, three, or more of the partitions may be annulled without vitiating the stability of the steady symmetric motion; but that if it be annulled through the whole of space, for all the partitions, the symmetric motion is unstable, and the rings shuffle themselves into perpetually varying relative positions, with average homogeneousness, like the ultimate molecules of a homogeneous liquid. 1 cannot see how, under these conditions, the "vitiating rearrangement" referred to at the end of $\S 20$ can be expected not to take place within the period of a wave or vibration. To suppose the overall diameter of each ring to lue very small in proportion to its average distances from neighbours, so that the crowd would be analogous rather to the molecules of a gas than to those of a liquid, would not help us to escape the vitiating rearrangement which would be analogous to that investigated by Maxwell in his admirable kinetic theory of the viscosity of gases. I am thus driven to admit, in conclusion, that the most favourable verdict I can ask for the propagation of laminar waves through a turbulently moving inviscid liquid is the Scottish verdict of not proven. 
Motion through a turbulently moving Inviscid Liquid. 353 Fig. 2.
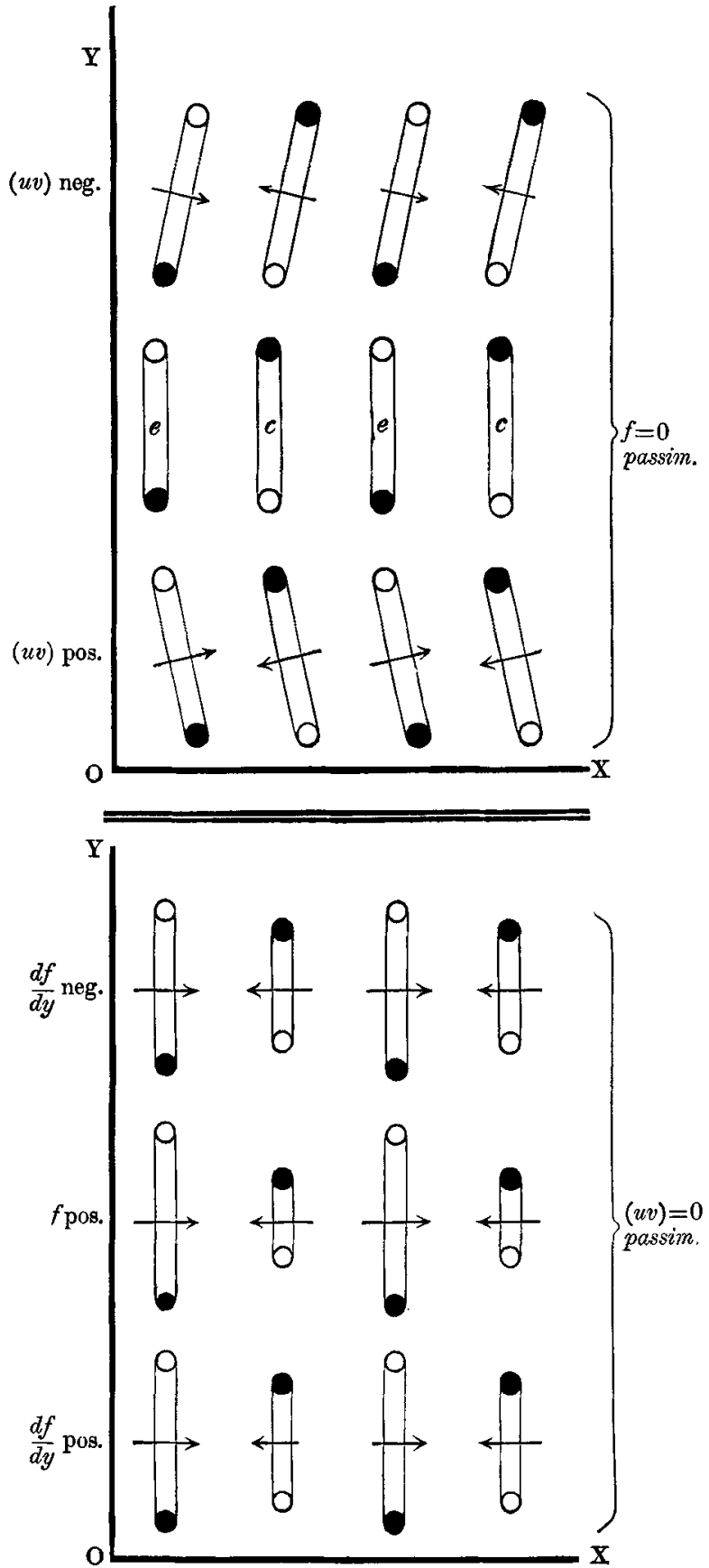

Here (uv) means an average of the kind described in the footnote on (46). 